\title{
Do que morrem os pacientes com tuberculose: causas múltiplas de morte de uma coorte de casos notificados e uma proposta de investigação de causas presumíveis
}

\author{
What are the causes of death of patients with \\ tuberculosis: multiple causes of death in a \\ cohort of cases and a research proposal of \\ presumed causes
}

\author{
Cuáles son las causas de muerte de pacientes \\ con tuberculosis: múltiples causas en una \\ cohorte de casos notificados y una \\ propuesta de investigación
}

\author{
Marli Souza Rocha 1 \\ Gisele Pinto de Oliveira 1 \\ Fernanda Pinheiro Aguiar 1 \\ Valéria Saraceni 2 \\ Rejane Sobrino Pinheiro 1
}

1 Instituto de Estudos em Saúde Coletiva, Universidade Federal do Rio de Janeiro, Rio de Janeiro, Brasil.

2 Secretaria Municipal de

Saúde do Rio de Janeiro, Rio

de Janeiro, Brasil.

Correspondência

R. S. Pinheiro

Instituto de Estudos em

Saúde Coletiva, Universidade Federal do Rio de Janeiro.

Pça. da Prefeitura da Cidade Universitária, antigo prédio do ETU, Rio de Janeiro, $R J$ 21944-970, Brasil.

rejanesp07@gmail.com

\begin{abstract}
The objective of this study was to analyze the multiple causes of death in a cohort of patients with tuberculosis (TB) and to introduce an investigation proposal death for TB from a list of presumable causes. We performed a probabilistic record linkage with the databases of the Information System for Notifiable Diseases (SINAN) 2006 and the Mortality Information System (SIM) 2006-2008. There were 825 deaths, of which $23 \%$ for death for $T B$, deaths due to $T B$ with $16 \%$ and $61 \%$ without mention of TB. Two hundred and fifteen (42.7\%) deaths occurred within the period of treatment, whose profile differed from the pattern of causes when TB was an associated cause, with high frequency of respiratory diseases, AIDS and ill-defined causes. We elaborated a proposal for correction of associated causes of death and an investigation proposal death for TB from a list of presumable causes. According to the proposal, 26 deaths could have modified the underlying cause. This study highlights the importance of record linkage to TB surveillance and improvement of information the SIM and SINAN.
\end{abstract}

Cause of Death; Tuberculosis; Mortality

\section{Resumo}

O objetivo deste trabalho foi analisar as causas múltiplas de morte de uma coorte de pacientes notificados com tuberculose (TB) e apresentar uma proposta de investigação de causas presumíveis. Realizou-se linkage probabilístico entre o Sistema de Informação de Agravos de Notificação (SINAN) 2006 e o Sistema de Informação sobre Mortalidade (SIM), 2006-2008. Ocorreram 825 mortes, das quais 23\% por TB, $16 \%$ com TB e $61 \%$ sem menção da TB. Duzentos e quinze (42,7\%) óbitos ocorreram antes do término do esquema básico de tratamento e não tinham menção da TB, cujo perfil foi distinto do padrão quando a TB era uma das causas associadas. A elevada frequência de doenças do aparelho respiratório, AIDS e causas mal definidas sugerem falha na qualidade da informação. Elaborouse proposta de correção das causas associadas no SIM e de investigação de óbito com base na relação de causas presumíveis. De acordo com a proposta, 26 óbitos poderiam ter a causa básica modificada. Este estudo destaca a gravidade do quadro da TB e a importância do linkage para a vigilância da TB e melhoria das informações do SIM e do SINAN.

Causas de Morte; Tuberculose; Mortalidade 


\section{Introdução}

O Brasil está entre os 22 países responsáveis por $80 \%$ dos casos de tuberculose (TB) no mundo, com cerca de 72 mil casos notificados e 4.500 mortes em 2011 1. O Município do Rio de Janeiro foi a segunda capital brasileira com a maior taxa de incidência e a primeira com a maior taxa de mortalidade nesse período (Departamento de Informática do SUS. Tabnet: tabulador na web. http://www2.datasus.gov.br/DATASUS/index. php, acessado em 15/Jul/2013).

O Sistema de Informação de Agravos de Notificação (SINAN) é o principal instrumento para o monitoramento dos casos de TB utilizado pela vigilância epidemiológica no país. No entanto, quando o óbito é o desfecho de interesse, o Sistema de Informação sobre Mortalidade (SIM) fornece informações importantes para o diagnóstico de saúde da população, que permitem a implementação de políticas e ações de saúde, visando a eliminar ou a diminuir situações de risco 2 .

A causa básica é utilizada para o cálculo do coeficiente de mortalidade por TB, um dos principais indicadores usados para o monitoramento e avaliação das ações de controle da TB, do diagnóstico ao tratamento. No entanto, esse indicador não reflete totalmente a contribuição da TB na mortalidade, uma vez que a TB ocorre como causa associada em uma grande proporção de óbitos em que a morte é atribuída a outras causas básicas 4. No Estado do Rio de Janeiro, em 2001, o coeficiente de mortalidade por TB quando identificada como causa básica foi de 7,1 por 100 mil habitantes. Quando calculado incluindo como causa associada, o coeficiente de mortalidade aumenta $40 \%$, indo para 10,0 por $100 \mathrm{mil}$ habitantes ${ }^{3}$.

Sendo a TB uma doença curável, a morte deveria ser um evento raro. Estudar as causas de morte dos pacientes de TB permite identificar fatores associados, grupos que apresentam maior risco e um conjunto de causas que podem aumentar a sua letalidade. Sendo assim, intervenções específicas podem ser adotadas para diminuir a ocorrência do evento ou o desfecho óbito na população.

Outro ponto a ser considerado é o fato de a tuberculose poder estar sub-registrada nos arquivos do SIM. Nesses casos, a vigilância de óbitos por TB exerce papel fundamental, permitindo identificar óbitos nos sistemas de informação e qualificá-los a partir da investigação ${ }^{4}$.

As causas presumíveis de óbito mascaram as prováveis causas de morte. Trata-se daquela morte cuja causa básica relaciona-se com a história clínica da TB, mas esta não consta na Declaração de Óbito (DO) por falhas no preenchimento ou na classificação. Ocorre apenas o registro do fato ocasionador do óbito, a causa terminal ou a lesão que sobreveio por último na sucessão de eventos que culminou com a morte. Dessa forma, ocultase a causa básica e impede-se a identificação do óbito por TB 2. Assim, a investigação dessas causas presumíveis permitirá melhorar a qualidade da informação real sobre a mortalidade por TB 5 .

O objetivo deste trabalho foi analisar as causas múltiplas de morte de uma coorte de casos notificados com TB segundo sexo, idade e tempo de sobrevida, apresentar uma proposta de inclusão da TB como causa de morte na DO em que a mesma não estiver mencionada e de investigação de óbitos com base em causas presumíveis de óbito por TB.

\section{Método}

Trata-se de um estudo longitudinal, não concorrente, de seguimento passivo por meio do método de linkage probabilístico entre as bases de dados do SINAN e do SIM. A população analisada é proveniente da coorte de pacientes residentes no Município do Rio de Janeiro diagnosticados e notificados com TB no SINAN, em 2006, no mesmo município. Os indivíduos que tiveram o diagnóstico antes de 2006 foram excluídos, assim como 74 pacientes que tiveram o diagnóstico modificado após a notificação, segundo informação da ficha de acompanhamento do SINAN. A remoção de duplicidades dos registros foi realizada previamente ao estudo, seguindo os critérios estabelecidos pelo Ministério da Saúde 6 . Os registros desses pacientes foram relacionados com os da base de dados do SIM do Estado do Rio de Janeiro, do período de 2006 a 2008.

Foram realizados cinco passos de blocagem, conforme recomendações apontadas em Coeli \& Camargo Jr. ${ }^{7}$. Para comparação automática dos registros entre as bases de dados foram usadas as variáveis nome, sexo e data de nascimento; as variáveis nome da mãe e endereço foram utilizadas para comparação visual na classificação dos links duvidosos ao final de cada passo de blocagem. Optou-se por realizar um procedimento mais conservador visando a aumentar a especificidade da classificação final do link como par verdadeiro.

Foram considerados óbitos por TB aqueles que tiveram causa básica ou causa associada (linhas A, B, C, D ou II) registradas com os códigos A15 a A19 da 10a revisão da Classificação Internacional de Doenças (CID-10). Foram ainda considerados óbitos com causa associada à $\mathrm{TB}$ aqueles que tinham código B20.0 (doença pe- 
lo HIV resultando em TB) como alguma causa de morte. Os óbitos referentes a sequelas de TB (B90) não foram considerados como caso de óbito pela doença.

Foram consideradas todas as causas de morte registradas na DO. Somente uma única causa associada por indivíduo foi contabilizada quando havia mais de uma menção à mesma categoria, e, consequentemente, ao mesmo capítulo da CID-10. Dessa forma, evitou-se a multiplicação da classificação ${ }^{8}$. A análise foi realizada segundo os capítulos da CID-10 e destacadas, nas tabelas, as subcategorias, ou categorias ou agrupamentos de categorias afins mais frequentes, especialmente quando se relacionavam com a TB ou com o sistema respiratório inferior. O percentual de cada capítulo, agrupamento, categoria ou subcategoria da tabela foi calculado sobre o total de óbitos, tanto para a causa básica quanto para a associada. Desse modo, o percentual de cada causa associada reflete a parcela dos óbitos que continham a referida causa. Utilizaram-se dois períodos de tempo como forma de estimar se o paciente estava em tratamento para TB à época da morte. O primeiro período iniciou a partir da data do diagnóstico até 180 dias, que corresponde ao regime de tratamento básico, e o segundo foi de 181 dias até o final do período de acompanhamento.

As análises foram realizadas segundo idade (menor que 15 anos, de 15 a 59 e 60 ou mais anos), sexo e o tempo de sobrevivência, definido a partir do número de dias transcorridos da data do diagnóstico no SINAN até a data de ocorrência do óbito no SIM, e categorizados em menor ou igual a 180 dias e maior de 180 dias, que corresponde ao esquema básico de tratamento.

A proposta de inclusão da TB como uma causa de morte foi baseada na ausência da doença na DO dos pacientes que se encontravam dentro do período de tempo relacionado ao esquema básico de tratamento (menos de 180 dias após o diagnóstico). A proposta de investigação foi elaborada para o subconjunto desses óbitos que possuíssem como causa básica uma causa presumível, uma vez que a tuberculose não teria sido identificada. Considerou-se, também, quando a TB era causa associada de óbito por lesão hepática tóxica (K71.9). A proposta baseou-se, ainda, na informação sobre exames laboratoriais (baciloscopia de escarro ou de outro material, histopatologia e cultura de escarro ou de outro material) presentes no SINAN, numa perspectiva mais conservadora.

O linkage probabilístico entre os registros foi realizado com o auxílio do software Reclink III (Camargo Jr. KR, Coeli CM. http://www.iesc.ufrj. br/reclink/RecLink_arquivos/RecLinkdl.html), e o software Epi Info 6.04 (Centers for Disease Control and Prevention, Atlanta, Estados Unidos) foi utilizado na análise descritiva e das causas múltiplas.

A base de dados do SINAN e SIM foram disponibilizadas, respectivamente, pela Secretaria Municipal de Saúde do Rio de Janeiro e pela Secretaria Estadual de Saúde do Rio de Janeiro. O trabalho foi aprovado pelo Comitê de Ética em Pesquisa do Instituto de Estudos em Saúde Coletiva, Universidade Federal do Rio de Janeiro (IESC/UFRJ), processo no 180.005/2012.

\section{Resultados}

A população do estudo foi constituída por 6.370 indivíduos notificados em 2006. A maioria era homens, com idades entre 15 e 59 anos.

Foram encontrados no SIM, entre 2006 e 2008, 825 (13\%) óbitos considerando todas as causas, sendo 190 (23\%) óbitos com causa básica TB, $132(16 \%)$ com causa associada TB e 503 (61\%) sem menção da TB na DO. A mediana de tempo até a morte foi de 150 dias (1-1.060 dias) considerando todos os óbitos, 47 dias (1-1.014 dias) para os óbitos de TB como causa básica, 98 (1-1.057 dias) para os de TB como causa associada e 246 (1-1.061 dias) para os sem menção da TB na DO.

Dos 190 óbitos que tiveram a TB como causa básica, houve 310 menções de causas associadas (Tabela 1). A maior frequência de menções foi para as doenças relacionadas com o aparelho respiratório (76,8\%), em especial, insuficiência respiratória, seguida de pneumonias e de doença pulmonar obstrutiva crônica. O segundo grupo com o maior número de menções foi o de sintomas, sinais e achados anormais de exames clínicos e de laboratório (23,7\%), seguido do grupo de doenças endócrinas e metabólicas com 22 menções (11,6\%; destes, $31,8 \%$ por diabetes e $50 \%$ por desnutrição). Destacaram-se, também, 6,8\% de transtornos mentais e comportamentais.

Para os 132 óbitos com causa associada à TB, aproximadamente $80 \%$ tinham AIDS como causa básica. O restante dos óbitos tinha como causa básica os tumores $(6,1 \%)$, sendo metade do aparelho digestivo, e as doenças do aparelho circulatório $(4,5 \%)$.

Dos 503 óbitos sem menção da TB na DO, $22,9 \%$ tinham AIDS como causa básica. As neoplasias e as doenças do aparelho respiratório surgiram em seguida $(16,7 \%$ e $13,5 \%$, respectivamente). Após esses, seguiam-se as causas externas $(11,7 \%)$, as doenças do aparelho circulatório $(10,1 \%)$ e as doenças do capítulo sintomas, sinais e achados anormais $(9,3 \%)$. 
Tabela 1

Perfil dos casos notificados com tuberculose (TB), dos óbitos por todas as causas, dos óbitos com a TB como causa básica e como causa associada e daqueles sem menção da TB na Declaração de Óbito (DO). Rio de Janeiro, Brasil, 2006 a 2008.

\begin{tabular}{|c|c|c|c|c|c|c|c|c|c|c|}
\hline \multirow[t]{2}{*}{ Variáveis } & \multicolumn{2}{|c|}{$\begin{array}{c}\text { Total de } \\
\text { notificados }\end{array}$} & \multicolumn{2}{|c|}{$\begin{array}{l}\text { Óbitos por todas } \\
\text { as causas }\end{array}$} & \multicolumn{2}{|c|}{$\begin{array}{l}\text { TB causa } \\
\text { básica }\end{array}$} & \multicolumn{2}{|c|}{$\begin{array}{l}\text { TB causa } \\
\text { associada }\end{array}$} & \multicolumn{2}{|c|}{$\begin{array}{c}\text { Óbitos sem menção } \\
\text { da TB na DO }\end{array}$} \\
\hline & $\mathrm{n}$ & $\%$ & $\mathrm{n}$ & $\%$ & $\mathrm{n}$ & $\%$ & $\mathrm{n}$ & $\%$ & $\mathrm{n}$ & $\%$ \\
\hline \multicolumn{11}{|l|}{ Sexo } \\
\hline Masculino & 4.086 & 64,1 & 567 & 68,7 & 124 & 65,3 & 86 & 65,2 & 357 & 71,0 \\
\hline Feminino & 2.284 & 35,9 & 258 & 31,3 & 66 & 34,7 & 46 & 34,8 & 146 & 29,0 \\
\hline \multicolumn{11}{|l|}{ Idade (anos) } \\
\hline$<15$ & 233 & 3,7 & 9 & 1,1 & 4 & 2,1 & 1 & 0,8 & 4 & 0,8 \\
\hline $15-59$ & 5.408 & 84,9 & 614 & 74,4 & 134 & 70,5 & 115 & 87 & 365 & 72,6 \\
\hline 60 ou mais & 720 & 11,3 & 202 & 24,5 & 52 & 27,4 & 16 & 12,2 & 134 & 26,6 \\
\hline \multicolumn{11}{|c|}{ Tempo de sobrevida (dias) } \\
\hline$\leq 180$ & 441 & 6,9 & 441 & 53,5 & 138 & 72,6 & 89 & 67,2 & 214 & 42,5 \\
\hline$>180$ & 5.929 & 93,1 & 384 & 46,5 & 52 & 27,4 & 43 & 32,8 & 289 & 57,5 \\
\hline Total & 6.370 & 100,0 & 825 & 13,0 & 190 & 23,0 & 132 & 16,0 & 503 & 61,0 \\
\hline
\end{tabular}

Os óbitos de TB como causa básica ou associada foram mais frequentes nos homens, seguindo o mesmo padrão da distribuição da doença: em torno de dois terços para homens e um terço para mulheres. Ocorreu diferença para os óbitos sem menção da TB na DO, destacando um pouco mais os homens $(71 \%)$ do que no padrão geral da doença. As causas de morte por sexo mostraram algumas diferenças entre homens e mulheres: nas causas básicas, homens tiveram maior frequência de doenças do aparelho circulatório (11,3\% para os homens $v s .4,5 \%$ para as mulheres) e de transtorno mental e comportamental (10,5\% para os homens $v s$. nenhuma ocorrência para as mulheres); nas causas associadas, os homens com mais neoplasias ( $9,3 \%$ para os homens vs. nenhuma para mulheres) e as mulheres com mais AIDS (76,7\% para os homens vs. $84,8 \%$ para as mulheres). Nos óbitos sem menção da TB na DO, destacam-se as causas externas para os homens $(15,1 \% v s .3,4 \%)$ e a AIDS $(19,6 \% v s .31,5 \%)$, e as neoplasias $(15,7 \%$ vs. $19,2 \%)$ para as mulheres (Tabela 2).

Quanto à idade, os óbitos com a TB como causa básica ou os sem menção da TB na DO foram mais frequentes nos idosos, se comparados com a distribuição etária dos casos notificados. Ao contrário, o padrão etário foi seguido para os óbitos com a TB como causa associada.

Quando a TB era a causa básica, os idosos se destacaram nas causas associadas, como septicemia, doenças do aparelho circulatório, doenças endócrinas e metabólicas, e neoplasias.
Aqueles com 15 a 59 anos destacaram-se nos óbitos por transtornos mentais e comportamentais (Tabela 3). Quando a TB era causa associada, as causas básicas diferiram consideravelmente entre os grupos etários. Para os de 15 a 59 anos, praticamente todos os óbitos tiveram como causa básica a AIDS (88,6\% vs. 25\% para os idosos), enquanto que os idosos morreram mais por neoplasias (25\%), doenças do aparelho respiratório $(12,5 \%)$ e doenças do aparelho digestivo (12,5\%). Nos óbitos sem menção da TB na DO, também houve acentuada diferença nas causas básicas entre os grupos etários. Para os com 15 a 59 anos, as causas mais frequentes foram: AIDS $(32,6 \%)$; causas externas $(14,8 \%)$; neoplasias $(13,7 \%)$, sendo que $32 \%$ eram de brônquios ou pulmões; e doenças do aparelho respiratório (9,3\%), sendo $55,9 \%$, pneumonias. Para os idosos, foram mais frequentes as doenças do aparelho respiratório $(25,4 \%)$, sendo $35,3 \%$ por pneumonias; as neoplasias $(24,6 \%)$, sendo $36,4 \%$ de brônquios ou pulmões; as doenças do aparelho circulatório $(15,7 \%)$. Destaca-se que aproximadamente $9 \%$ dos óbitos foram por sintomas, sinais e achados anormais.

Nos dois períodos analisados (tempo de sobrevida menor ou maior que 180 dias após o diagnóstico), houve diferença entre as causas de morte, com exceção dos óbitos com causa associada à TB (Tabela 4).

Considerando o período de tempo entre o diagnóstico e a morte, mais de dois terços dos óbitos com menção da TB na DO ocorreram 
Causas associadas quando a tuberculose (TB) era causa básica na Declaração de Óbito (DO), segundo sexo, grupo etário e períodos de sobrevida. Rio de Janeiro, Brasil, 2006 a 2008.

\begin{tabular}{|c|c|c|c|c|c|c|c|c|c|c|c|c|c|c|}
\hline \multirow[t]{4}{*}{ Causas associadas de óbito } & \multicolumn{14}{|c|}{ TB como causa básica de morte (190) } \\
\hline & \multirow{2}{*}{\multicolumn{2}{|c|}{ Total }} & \multicolumn{4}{|c|}{ Sexo } & \multicolumn{4}{|c|}{ Idade (anos) } & \multicolumn{4}{|c|}{ Sobrevida (dias) } \\
\hline & & & \multicolumn{2}{|c|}{ Masculino } & \multicolumn{2}{|c|}{ Feminino } & \multicolumn{2}{|c|}{$15-59$} & \multicolumn{2}{|c|}{$\geq 60$} & \multicolumn{2}{|c|}{$\leq 180$} & \multicolumn{2}{|c|}{$>180$} \\
\hline & $\mathbf{n}$ & $\%$ & $\mathbf{n}$ & $\%$ & n & $\%$ & $\mathbf{n}$ & $\%$ & $\mathbf{n}$ & $\%$ & $\mathbf{n}$ & $\%$ & $\mathbf{n}$ & $\%$ \\
\hline Doenças infecto-parasitárias (A00-B99) & 29 & 15,3 & 20 & 16,1 & 9 & 13,6 & 13 & 9,7 & 14 & 26,9 & 23 & 16,7 & 6 & 11,5 \\
\hline \multicolumn{15}{|l|}{ Doença pelo vírus imunodeficiência humana } \\
\hline (B20-B24) & 0 & & 0 & & 0 & & 0 & & 0 & & 0 & & 0 & \\
\hline Septicemia (A40-A41) & 25 & & 17 & & 8 & & 10 & & 13 & & 20 & & 5 & \\
\hline Neoplasias [tumores] (C00-D48) & 6 & 3,2 & 5 & 4,0 & 1 & 1,5 & 3 & 2,2 & 3 & 5,8 & 6 & 4,3 & 0 & 0,0 \\
\hline Neoplasia brônquios e pulmões (C349) & 0 & & 0 & & 0 & & 0 & & 0 & & 0 & & 0 & \\
\hline Neoplasias órgãos digestivos (C15-C26) & 2 & & 2 & & 0 & & 1 & & 1 & & 2 & & 0 & \\
\hline Doenças do sangue (D50-D89) & 1 & 0,5 & 1 & 0,8 & 0 & 0,0 & 1 & 0,7 & 0 & 0,0 & 1 & 0,7 & 0 & 0,0 \\
\hline Doenças endócrinas (E00-E90) & 22 & 11,6 & 14 & 11,3 & 8 & 12,1 & 13 & 9,7 & 8 & 15,4 & 16 & 11,6 & 6 & 11,5 \\
\hline Diabetes mellitus (E10-E14) & 7 & & 2 & & 5 & & 3 & & 4 & & 6 & & 1 & \\
\hline Desnutrição (E40-E46) & 11 & & 9 & & 2 & & 6 & & 4 & & 8 & & 3 & \\
\hline Transtornos mentais e comportamentais (F00-F99) & 13 & 6,8 & 13 & 10,5 & 0 & 0,0 & 10 & 7,5 & 3 & 5,8 & 9 & 6,5 & 4 & 7,7 \\
\hline Transtornos mentais uso de álcool (F102) & 10 & & 10 & & 0 & & 7 & & 3 & & 7 & & 3 & \\
\hline Transtornos mentais uso de fumo (F172) & 3 & & 3 & & 0 & & 3 & & 0 & & 2 & & 1 & \\
\hline Doenças do sistema nervoso (G00-G99) & 3 & 1,6 & 3 & 2,4 & 0 & 0,0 & 3 & 2,2 & 0 & 0,0 & 3 & 2,2 & 0 & 0,0 \\
\hline Doenças aparelho circulatório (I00-199) & 17 & 8,9 & 14 & 11,3 & 3 & 4,5 & 6 & 4,5 & 9 & 17,3 & 16 & 11,6 & 1 & 1,9 \\
\hline Cardiopatia pulmonar (I279) & 1 & & 1 & & 0 & & 1 & & 0 & & 0 & & 1 & \\
\hline Doenças do aparelho respiratório (J00-J99) & 146 & 76,8 & 93 & 75,0 & 53 & 80,3 & 103 & 76,9 & 42 & 80,8 & 109 & 79,0 & 37 & 71,2 \\
\hline Pneumonias (J12-J18) & 20 & & 13 & & 7 & & 12 & & 8 & & 15 & & 5 & \\
\hline \multicolumn{15}{|l|}{ Restante aparelho respiratório (J40-J47, J81, } \\
\hline J90-J94) & 13 & & 8 & & 5 & & 7 & & 5 & & 9 & & 4 & \\
\hline Insuficiência respiratória (J96) & 100 & & 63 & & 37 & & 74 & & 26 & & 76 & & 24 & \\
\hline Outros transtornos respiratórios (J98) & 8 & & 6 & & 2 & & 6 & & 2 & & 5 & & 3 & \\
\hline Doenças do aparelho digestivo (K00-K93) & 16 & 8,4 & 12 & 9,7 & 4 & 6,1 & 9 & 6,7 & 7 & 13,5 & 14 & 10,1 & 2 & 3,8 \\
\hline \multicolumn{15}{|l|}{ Doença hepática tóxica com hepatite não } \\
\hline classificada em outra parte (K716) & 5 & & 3 & & 2 & & 1 & & 4 & & 4 & & 1 & \\
\hline Doenças da pele e tecido subcutâneo (L00-L99) & 1 & 0,5 & 0 & 0,0 & 1 & 1,5 & 0 & 0,0 & 1 & 1,9 & 0 & 0,0 & 1 & 1,9 \\
\hline Doenças sistema osteomuscular (M00-M99) & 0 & 0,0 & 0 & 0,0 & 0 & 0,0 & 0 & 0,0 & 0 & 0,0 & 0 & 0,0 & 0 & 0,0 \\
\hline Doenças aparelho geniturinário (N00-N99) & 7 & 3,7 & 5 & 4,0 & 2 & 3,0 & 5 & 3,7 & 2 & 3,8 & 5 & 3,6 & 2 & 3,8 \\
\hline \multicolumn{15}{|l|}{ Malformação congênita, deformidades e } \\
\hline anomalias congênitas (Q00-Q99) & 0 & 0,0 & 0 & 0,0 & 0 & 0,0 & 0 & 0,0 & 0 & 0,0 & 0 & 0,0 & 0 & 0,0 \\
\hline Sintomas, sinais e achados anormais (R00-R99) & 45 & 23,7 & 27 & 21,8 & 18 & 27,3 & 32 & 23,9 & 11 & 21,2 & 35 & 25,4 & 10 & 19,2 \\
\hline \multicolumn{15}{|l|}{ Sintomas e sinais aparelhos circulatório e } \\
\hline respiratório (R00-R09) & 16 & & 11 & & 5 & & 14 & & 2 & & 11 & & 5 & \\
\hline Hemorragias de vias respiratórias (R04) & 4 & & 3 & & 1 & & 4 & & 0 & & 3 & & 1 & \\
\hline Hemoptise (R042) & 4 & & 7 & & 4 & & 9 & & 2 & & 3 & & 1 & \\
\hline Sintomas e sinais gerais (R50-R69) & 28 & & 3 & & 1 & & 4 & & 0 & & 23 & & 5 & \\
\hline Parada respiratória (R092) & 11 & & 0 & & 1 & & 0 & & 1 & & 7 & & 4 & \\
\hline Causas mal definidas (R95-R99) & 1 & & 13 & & 15 & & 18 & & 8 & & 1 & & 0 & \\
\hline Caquexia (R64) & 12 & & 7 & & 5 & & 9 & & 3 & & 8 & & 4 & \\
\hline Lesões, envenenamento e algumas outras & & & & & & & & & & & & & & \\
\hline consequências de causas externas (S00-T98) & 2 & 1,1 & 1 & 0,8 & 1 & 1,5 & 1 & 0,7 & 0 & 0,0 & 2 & 1,4 & 0 & 0,0 \\
\hline Causas externas morbidade e mortalidade & & & & & & & & & & & & & & \\
\hline (V01-Y98) & 2 & 1,1 & 0 & 0,0 & 2 & 3,0 & 0 & 0,0 & 1 & 1,9 & 1 & 0,7 & 1 & 1,9 \\
\hline Total de menções & 310 & NC & 208 & NC & 102 & NC & 182 & NC & 95 & NC & 240 & NC & 70 & NC \\
\hline
\end{tabular}

NC: não calculado.

Notas: capítulos da CID-10 que não tinham óbitos foram excluídos. As porcentagens são calculadas em relação ao número de óbitos pela causa básica correspondente. 
Causas básicas quando a tuberculose (TB) era causa associada segundo sexo, grupo etário e períodos de sobrevida. Rio de Janeiro, Brasil, 2006 a 2008.

\begin{tabular}{|c|c|c|c|c|c|c|c|c|c|c|c|c|c|c|}
\hline \multirow[t]{4}{*}{ Causas básicas de óbito } & \multicolumn{14}{|c|}{ TB como causa associada de morte (132) } \\
\hline & \multirow{2}{*}{\multicolumn{2}{|c|}{ Total }} & \multicolumn{4}{|c|}{ Sexo } & \multicolumn{4}{|c|}{ Idade (anos) * } & \multicolumn{4}{|c|}{ Sobrevida (dias) } \\
\hline & & & \multicolumn{2}{|c|}{ Masculino } & \multicolumn{2}{|c|}{ Feminino } & \multicolumn{2}{|c|}{$15-59$} & \multicolumn{2}{|c|}{$\geq 60$} & \multicolumn{2}{|c|}{$\leq 180$} & \multicolumn{2}{|c|}{$>180$} \\
\hline & n & $\%$ & $\mathbf{n}$ & $\%$ & n & $\%$ & $\mathbf{n}$ & $\%$ & n & $\%$ & $\mathbf{n}$ & $\%$ & n & $\%$ \\
\hline Doenças infecto-parasitárias (A00-B99) & 107 & 81,1 & 68 & 79,1 & 39 & 84,8 & 102 & 88,6 & 4 & 25,0 & 72 & 80,9 & 35 & 81,4 \\
\hline \multicolumn{15}{|l|}{ Doença pelo vírus imunodeficiência } \\
\hline humana (B20-B24) & 105 & & 66 & & 39 & & 100 & & 4 & & 70 & & 35 & \\
\hline Septicemia (A40-A41) & 0 & & & & & & & & & & 0 & & 0 & \\
\hline Neoplasias [tumores] (C00-D48) & 8 & 6,1 & 8 & 9,3 & 0 & 0,0 & 4 & 3,5 & 4 & 25,0 & 6 & 6,7 & 2 & 4,7 \\
\hline \multicolumn{15}{|l|}{ Neoplasia brônquios e pulmões } \\
\hline (C349) & 0 & & & & & & & & & & 0 & & 0 & \\
\hline \multicolumn{15}{|l|}{ Neoplasias órgãos digestivos } \\
\hline$(\mathrm{C} 15-\mathrm{C} 26)$ & 4 & & 4 & & 0 & & 2 & & 2 & & 3 & & 1 & \\
\hline Doenças do sangue (D50-D89) & 1 & 0,8 & 0 & 0,0 & 1 & 2,2 & 0 & 0,0 & 1 & 6,3 & 1 & 1,1 & 0 & 0,0 \\
\hline Doenças endócrinas (E00-E90) & 2 & 1,5 & 2 & 2,3 & 0 & 0,0 & 1 & 0,9 & 1 & 6,3 & 2 & 2,2 & 0 & 0,0 \\
\hline \multicolumn{15}{|l|}{ Doenças do aparelho circulatório } \\
\hline (100-199) & 6 & 4,5 & 5 & 5,8 & 1 & 2,2 & 5 & 4,4 & 1 & 6,3 & 2 & 2,2 & 4 & 9,3 \\
\hline Cardiopatia pulmonar (I279) & 0 & & & & & & & & & & 0 & & 0 & \\
\hline \multicolumn{15}{|l|}{ Doenças do aparelho respiratório } \\
\hline (J00-J99) & 2 & 1,5 & 1 & 1,2 & 1 & 2,2 & 0 & 0,0 & 2 & 12,5 & 1 & 1,1 & 1 & 2,3 \\
\hline Pneumonias (J12-J18) & 0 & & & & & & & & & & 0 & & 0 & \\
\hline \multicolumn{15}{|l|}{ Restante aparelho respiratório } \\
\hline (J40-J47, J81, J90-J94) & 1 & & 0 & & 1 & & 0 & & 1 & & 1 & & 0 & \\
\hline Insuficiência respiratória (J96) & 0 & & & & & & & & & & 0 & & 0 & \\
\hline \multicolumn{15}{|l|}{ Outros transtornos respiratórios } \\
\hline (J98) & 0 & & & & & & & & & & 0 & & 0 & \\
\hline \multicolumn{15}{|l|}{ Doenças do aparelho digestivo } \\
\hline (K00-K93) & 4 & 3,0 & 2 & 2,3 & 2 & 4,3 & 2 & 1,8 & 2 & 12,5 & 3 & 3,4 & 1 & 2,3 \\
\hline Doença hepática tóxica (K716) & 2 & & 1 & & 1 & & 1 & & 1 & & 2 & & 0 & \\
\hline Doenças da pele (LOO-L99) & 0 & 0,0 & & & & & & & & & 0 & 0,0 & 0 & 0,0 \\
\hline \multicolumn{15}{|l|}{ Doenças do sistema osteomuscular } \\
\hline (M00-M99) & 2 & 1,5 & 0 & 0,0 & 2 & 4,3 & 1 & 0,9 & 1 & 6,3 & 2 & 2,2 & 0 & 0,0 \\
\hline Total de menções & 132 & 100,0 & 86 & 100,0 & 46 & 100,0 & 115 & 100,0 & 16 & 100,0 & 89 & 100,0 & 43 & 100,0 \\
\hline
\end{tabular}

* Menores de 15 anos: um óbito por doença pelo vírus da imunodeficiência humana.

Nota: capítulos da CID-10 que não tinham óbitos foram excluídos.

durante o período de 180 dias: $72,6 \%$ dos óbitos com causa básica TB e 67,4\% com causa associada à TB. Para os óbitos sem menção da TB na DO, esse percentual foi de $42,5 \%$.

Observou-se que, nos casos em que a TB foi a causa básica de óbito, as causas associadas mais comuns estavam relacionadas, direta ou indiretamente, com a doença ou com a sua gravidade. Para os óbitos ocorridos em menos de 180 dias, destacaram-se, ainda, as doenças do aparelho circulatório $(11,6 \%)$, do aparelho digestivo (10,1\%), sendo que $28,6 \%$ destas eram doenças hepáticas, e as neoplasias $(4,3 \%)$, que podem ter contribuído para o agravamento do quadro da doença. Quando a TB foi registrada como causa associada, a causa básica mais frequente, em torno de $80 \%$, foi a AIDS, nos dois períodos.

Para os óbitos sem menção da TB na DO, a distribuição das causas básicas de óbito diferiu entre os períodos (Tabela 4). Nos óbitos ocorridos em até 180 dias após o diagnóstico, aproximadamente um terço foi por AIDS (31,3\%), seguidos das neoplasias (15,9\%, sendo $47 \%$ delas de brônquios ou pulmões) e doenças do aparelho respiratório (15,4\%), e dos sinais e sintomas e achados anormais (13,6\%). Nos óbitos ocorridos após 180 
Causas básicas quando não havia menção da tuberculose (TB) na Declaração de Óbito (DO) segundo sexo, grupo etário e períodos de sobrevida. Rio de Janeiro, Brasil, 2006 a 2008.

\begin{tabular}{|c|c|c|c|c|c|c|c|c|c|c|c|c|c|c|}
\hline \multirow[t]{4}{*}{ Causas básicas de óbito } & \multicolumn{14}{|c|}{ Óbitos sem menção da TB (503) } \\
\hline & \multirow{2}{*}{\multicolumn{2}{|c|}{ Total }} & \multicolumn{4}{|c|}{ Sexo } & \multicolumn{4}{|c|}{ Idade (anos) * } & \multicolumn{4}{|c|}{ Sobrevida (dias) } \\
\hline & & & \multicolumn{2}{|c|}{ Masculino } & \multicolumn{2}{|c|}{ Feminino } & \multicolumn{2}{|c|}{$15-59$} & \multicolumn{2}{|c|}{$\geq 60$} & \multicolumn{2}{|c|}{$\leq 180$} & \multicolumn{2}{|c|}{$>180$} \\
\hline & $\mathbf{n}$ & $\%$ & $\mathbf{n}$ & $\%$ & $\mathbf{n}$ & $\%$ & $\mathbf{n}$ & $\%$ & $\mathbf{n}$ & $\%$ & $\mathbf{n}$ & $\%$ & $\mathbf{n}$ & $\%$ \\
\hline Doenças infecto-parasitárias (A00-B99) & 127 & 25,2 & 76 & 21,3 & 51 & 34,9 & 119 & 32,6 & 7 & 5,2 & 67 & 31,3 & 60 & 20,8 \\
\hline \multicolumn{15}{|l|}{ Doença pelo vírus imunodeficiência } \\
\hline humana (B20-B24) ** & 115 & & 70 & & 46 & & 111 & & 3 & & 61 & & 54 & \\
\hline Septicemia (A40-A41) & 7 & & 2 & & 5 & & 4 & & 3 & & 5 & & 2 & \\
\hline Neoplasias e tumores (C00-D48) & 84 & 16,7 & 56 & 15,7 & 28 & 19,2 & 50 & 13,7 & 33 & 24,6 & 34 & 15,9 & 50 & 17,3 \\
\hline \multicolumn{15}{|l|}{ Neoplasias brônquios e pulmões } \\
\hline (C349) & 28 & & 17 & & 11 & & 16 & & 12 & & 16 & & 12 & \\
\hline \multicolumn{15}{|l|}{ Neoplasias órgãos digestivos } \\
\hline$(\mathrm{C} 15-\mathrm{C} 26)$ & 13 & & 11 & & 2 & & 9 & & 4 & & 1 & & 12 & \\
\hline Doenças do sangue (D50-D89) & 5 & 1,0 & 4 & 1,1 & 1 & 0,7 & 4 & 1,1 & 1 & 0,7 & 3 & 1,4 & 2 & 0,7 \\
\hline Doenças endócrinas (E00-E90) & 21 & 4,2 & 16 & 4,5 & 5 & 3,4 & 12 & 3,3 & 9 & 6,7 & 9 & 4,2 & 12 & 4,2 \\
\hline Diabetes mellitus (E10-E14) & 13 & & 8 & & 5 & & 7 & & 6 & & 8 & & 5 & \\
\hline Desnutrição (E40-E46) & 3 & & 3 & & 0 & & 2 & & 1 & & 0 & & 3 & \\
\hline \multicolumn{15}{|l|}{ Transtornos mentais e } \\
\hline comportamentais (F00-F99) & 4 & 0,8 & 2 & 0,6 & 2 & 1,4 & 2 & 0,5 & 2 & 1,5 & 0 & 0,0 & 4 & 1,4 \\
\hline Transtornos mentais uso álcool (F102) & 2 & & 1 & & 1 & & 1 & & 1 & & 0 & & 2 & \\
\hline Transtornos mentais uso fumo (F172) & 1 & & 1 & & 0 & & 0 & & 1 & & 0 & & 1 & \\
\hline \multicolumn{15}{|l|}{ Doenças do sistema nervoso } \\
\hline (G00-G99) & 6 & 1,2 & 4 & 1,1 & 2 & 1,4 & 5 & 1,4 & 1 & 0,7 & 5 & 2,3 & 1 & 0,3 \\
\hline Doenças aparelho circulatório (100-199) & 51 & 10,1 & 37 & 10,4 & 14 & 9,6 & 30 & 8,2 & 21 & 15,7 & 16 & 7,5 & 35 & 12,1 \\
\hline Cardiopatia pulmonar (I279) & 0 & & & & & & & & & & 0 & & 0 & \\
\hline \multicolumn{15}{|l|}{ Doenças aparelho respiratório } \\
\hline (J00-J99) & 68 & 13,5 & 48 & 13,4 & 20 & 13,7 & 34 & 9,3 & 34 & 25,4 & 33 & 15,4 & 35 & 12,1 \\
\hline Pneumonias (J12-J18) & 31 & & 19 & & 12 & & 19 & & 12 & & 17 & & 14 & \\
\hline Restante aparelho respiratório (J40- & & & & & & & & & & & & & & \\
\hline J47,J81,J90-J94) & 21 & & 16 & & 5 & & 4 & & 17 & & 10 & & 11 & \\
\hline Insuficiência respiratória (J96) & 3 & & 2 & & 1 & & 1 & & 2 & & 1 & & 2 & \\
\hline Outros transtornos respiratórios & & & & & & & & & & & & & & \\
\hline (J98) & 11 & & 9 & & 2 & & 8 & & 3 & & 5 & & 6 & \\
\hline Doenças do aparelho digestivo & & & & & & & & & & & & & & \\
\hline (K00-К93) & 16 & 3,2 & 15 & 4,2 & 1 & 0,7 & 12 & 3,3 & 4 & 3,0 & 2 & 0,9 & 14 & 4,8 \\
\hline Doenças sistema osteomuscular & & & & & & & & & & & & & & \\
\hline (M00-M99) & 2 & 0,4 & 2 & 0,6 & 0 & 0,0 & 1 & 0,3 & 1 & 0,7 & 0 & 0,0 & 2 & 0,7 \\
\hline Doenças aparelho geniturinário & & & & & & & & & & & & & & \\
\hline (NOO-N99) & 12 & 2,4 & 8 & 2,2 & 4 & 2,7 & 7 & 1,9 & 5 & 3,7 & 3 & 1,4 & 9 & 3,1 \\
\hline Malformação congênita (Q00-Q99) & 1 & 0,2 & 1 & 0,3 & 0 & 0,0 & 1 & 0,3 & 0 & 0,0 & 1 & 0,5 & 0 & 0,0 \\
\hline Sintomas, sinais anormais (R00-R99) & 47 & 9,3 & 34 & 9,5 & 13 & 8,9 & 34 & 9,3 & 13 & 9,7 & 29 & 13,6 & 18 & 6,2 \\
\hline Hemoptise (R042) & 0 & & 3 & & 1 & & 3 & & 1 & & 0 & & 0 & \\
\hline Sintomas e sinais gerais (R50-R69) & 2 & & 0 & & 0 & & 0 & & 0 & & 0 & & 2 & \\
\hline Parada respiratória (R092) & 4 & & 29 & & 12 & & 31 & & 10 & & 4 & & 0 & \\
\hline Causas mal definidas (R95-R99) & 41 & & 2 & & 0 & & 0 & & 2 & & 25 & & 16 & \\
\hline Caquexia (R64) & 0 & & 0 & & 0 & & 0 & & 0 & & 0 & & 0 & \\
\hline Causas externas (V01-Y98) & 59 & 11,7 & 54 & 15,1 & 5 & 3,4 & 54 & 14,8 & 3 & 2,2 & 12 & 5,6 & 47 & 16,3 \\
\hline Total de menções & 503 & 100,0 & 357 & 100,0 & 146 & 100,0 & 365 & 100,0 & 134 & 100,0 & 214 & 100,0 & 289 & 100,0 \\
\hline
\end{tabular}

* Menores de 15 anos: quatro mortes (doença pelo vírus da imunodeficiência humana: 1, neoplasia: 1, causas externas: 2);

** Nenhum óbito registrado como B20.0 como causa básica ou associada.

Nota: capítulos da CID-10 que não tinham óbitos foram excluídos. 
dias do diagnóstico, a AIDS também foi a causa básica mais frequente $(20,8 \%)$, porém de maneira menos expressiva que no período anterior, seguidos, também, das neoplasias (17,3\%, sendo $24 \%$ delas referentes a brônquios e pulmões e $24 \%$ ao aparelho digestivo), das causas externas $(16,3 \%)$, das doenças do aparelho respiratório $(12,1 \%)$ e do aparelho circulatório $(12,1 \%)$.

O fluxograma da Figura 1 apresenta uma proposta para a inclusão da TB como causa de morte, para posterior reclassificação da causa básica, assim como critérios para investigação de causas presumíveis de óbito por TB.

As seguintes causas básicas de óbitos foram consideradas como presumíveis de óbito por TB: doença do aparelho digestivo (lesão hepática tóxica - K71), neoplasias (aparelho respiratório inferior - C34.9, C38.3, C38.4), doenças do aparelho respiratório inferior (J15.9, J18.0, J18.1, J18.9, J43.9, J44.9, J81, J90, J94.2, J96.0, J96.9, J98.4, J98.8), septicemia (A41.9) e sintomas, sinais e achados anormais (R00-R09, R50-R69, R96-R99).

A investigação seria realizada baseando-se na revisão de prontuários e no livro de registros de pacientes e acompanhamento de casos de TB da unidade de saúde onde o paciente era tratado, na unidade onde ocorreu o óbito, nos laboratórios para encontrar resultado de exames de diagnóstico, ou mesmo no domicílio, com o objetivo de coletar informações que possam auxiliar no encerramento da investigação. Para a investigação, deve-se buscar informações importantes, como resultado de exames (baciloscopia, cultura, histopatologia, radiografia de tórax, sorologia para HIV), relato em prontuários sobre uso de medicamentos, se havia resistência a alguma das medicações, se houve suspeita de TB no momento da internação, trajetória do paciente até a morte, histórico do tratamento, descrição das causas de morte.

Para o grupo de óbitos sem menção da TB na DO e ocorridos em menos de 180 dias, 63 (29,3\%) deveriam tê-la como causa de morte por apresentarem resultado de exame positivo para a $\mathrm{TB}$ no SINAN. Entretanto, esse número poderia ser maior, já que para 53 (24,7\%) óbitos do grupo não foi realizado nenhum exame. Ressalta-se que alguns desses, após uso do algoritmo de classificação, poderiam ter a TB como causa básica.

Ainda considerando os óbitos sem menção da TB na DO que estavam dentro do período estimado para tratamento, havia 79 com causa básica pertencente à lista de causas presumíveis. Vinte e seis (32,9\%) desses óbitos tinham resultado positivo para TB e poderiam ser investigados: $7,7 \%$ de septicemias ( $\mathrm{n}=2$ ), $15,4 \%$ de neoplasias do aparelho respiratório inferior $(n=4), 38,5 \%$ de doenças relacionadas ao aparelho respiratório
( $\mathrm{n}=10), 7,7 \%$ de lesões hepáticas tóxicas $(\mathrm{n}=2)$ e $30,7 \%$ por causas mal definidas $(n=8)$.

\section{Discussão}

A TB parece ser a causa principal de morte para os pacientes notificados com a doença. Aproximadamente um quarto morreu em decorrência da TB no período de dois anos após o diagnóstico, proporção esta que aumentaria para $31 \%$ caso reduzíssemos o período de seguimento para 180 dias ou se considerássemos a parcela expressiva dos óbitos sem menção da TB na DO que parece estar direta ou indiretamente relacionada à doença. Isso aponta para a magnitude do problema, embora exista tratamento eficaz amplamente disponibilizado na rede pública de saúde e de ser um óbito considerado evitável 9.

As causas de morte por sexo não diferiram do padrão geral. O que não foi observado quanto à idade. Nos dois períodos analisados não houve diferença entre as causas de morte, com exceção dos óbitos sem menção da TB na DO, nos quais as causas externas surgiram como a terceira causa mais frequente na sobrevida acima de 180 dias.

Nos óbitos com causa básica TB, a presença de septicemia, insuficiência respiratória, pneumonia, sintomas e sinais relativos ao aparelho respiratório (hemoptise, hemorragia de vias respiratórias, parada respiratória) como causas associadas refletem a gravidade da situação devido à presença de afecções respiratórias e quadros terminais, resultando na hospitalização seguida de morte, muitas vezes nas primeiras 24 horas 10 . A morte em função da doença não parece estar exclusivamente relacionada com populações reconhecidamente vulneráveis. Os agravos associados a piores desfechos (diabetes, transtorno mental e AIDS), que poderiam ter contribuído diretamente para o óbito, foram menos expressivos 11. A gravidade dos casos pode estar relacionada com outras comorbidades, como doença do aparelho circulatório, doenças do aparelho digestivo e neoplasias, assim como atraso no diagnóstico e falhas no tratamento e acompanhamento dos pacientes.

Mesmo com a presença de uma variável específica na ficha de notificação/investigação da TB, são poucas as afecções e agravos enumerados, além de não considerar a gravidade da doença e o campo "outras" ser aberto. Adicionalmente, a presença de comorbidades pode estar subestimada, porque estas informações são baseadas em uma entrada opcional na ficha de notificação 11. Ainda assim, Reis-Santos et al. 11 demostraram que indivíduos com duas ou mais 
Fluxograma de critérios para alteração da causa associada no Sistema de Informação sobre Mortalidade (SIM) e para a investigação de óbitos presumíveis por tuberculose (TB).

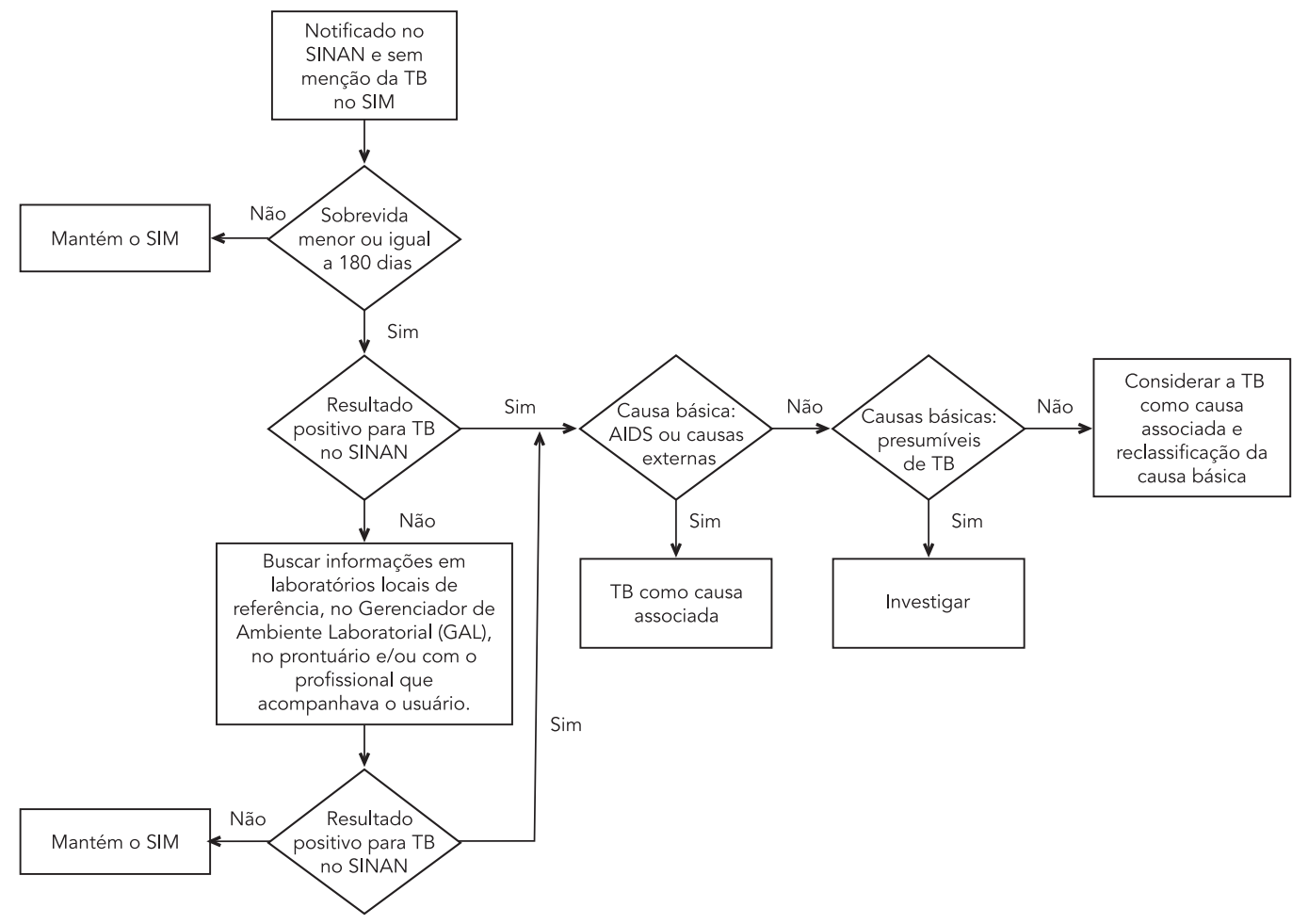

SINAN: Sistema de Informação de Agravos de Notificação.

comorbidades tendem a ser mais idosos, do sexo feminino e apresentarem maior mortalidade por outras causas.

Ao contrário do esperado, uma parcela considerável dos óbitos ocorreu dentro do período de tratamento e não teve a TB registrada pelo menos como causa associada. Algumas situações poderiam explicar a ausência de registro da doença: pacientes que iniciaram o tratamento empírico para $\mathrm{TB}$, mas que, posteriormente, tiveram o diagnóstico descartado e não foi feito o encerramento por mudança de diagnóstico; o desconhecimento da TB por parte do médico que preencheu a declaração de óbito; e, por último, a falta de investigação do óbito por TB quando não há confirmação diagnóstica laboratorial 10.

A distribuição das causas dos óbitos sem menção da TB na DO não seguiu o padrão geral de mortalidade da população do Município do Rio de Janeiro. Para os pacientes da coorte do estudo que foram a óbito, $25 \%$ das mortes foram por AIDS, 20\% por doenças relacionadas com o aparelho respiratório (as propriamente ditas e as neoplasias de brônquios e pulmões) e quase $10 \%$ por causas mal definidas, sugerindo a simultaneidade da TB com estas afecções. Possivelmente, esses eram óbitos diretamente relacionados com a TB, que não foi considerada no momento da internação, talvez por desconhecimento do padrão da doença; ou por se tratar de grupo populacional de difícil diagnóstico, como idosos; ou por falta de acesso a exames para confirmação da doença. Essa hipótese ganha destaque quando se identifica que o padrão de causas de morte é bem diferente quando a TB está registrada como causa associada 12,13,14.

Um percentual considerável de óbitos sem menção de TB na DO ocorreu por neoplasias. Em torno de um terço foi de neoplasias do aparelho respiratório, para ambos os sexos, tanto para os adultos quanto para os idosos. Esses percentuais foram superiores à distribuição das neoplasias 
no município, em que as de brônquios e pulmões corresponderam a $13,6 \%$ do total de neoplasias, em 2006 (Departamento de Informática do SUS. Tabnet: tabulador na web. http://www2.datasus. gov.br/DATASUS/index.php, acessado em 15/ Jul/2013). Os sinais e sintomas evidentes podem confundir o diagnóstico, até mesmo com imagens radiológicas compatíveis 15. Por isso, exames bacteriológicos, como a baciloscopia e a cultura, acopladas ou não à broncoscopia ou à biopsia, são exames que devem ser utilizados para a confirmação diagnóstica. $\mathrm{O}$ fato do percentual de neoplasias de brônquios e pulmões ter sido maior entre os mais velhos e entre as mulheres, diferente do perfil dessa doença na população, reforça a hipótese de erro no diagnóstico do óbito na rede hospitalar, uma vez que é mais difícil diagnosticar a TB em idosos pelas diversas comorbidades e por não ser a TB a primeira hipótese em mulheres, dado que é mais prevalente em homens 16.

O elevado percentual de óbitos por TB aponta para a deficiência da captação e do acompanhamento dos casos na atenção básica 17,18 e de amplas lacunas na comunicação entre a rede hospitalar, a de urgência e emergência e o programa de controle da TB. Os casos graves, ao darem entrada nas emergências, seguem a óbito, sem uma oportuna avaliação da história atual e pregressa do paciente ou da confirmação do diagnóstico. No entanto, a baciloscopia é um exame disponível na rede e, em um município com alta incidência de TB, é importante que os profissionais de saúde estejam atentos para a ocorrência da doença.

Os resultados sugerem que as doenças do aparelho circulatório estejam relacionadas com a precocidade do óbito do paciente com $\mathrm{TB}$, dado o mais elevado percentual de mortes que mencionam estas causas dentro do período de tratamento. Esse fato pode ocorrer pelo possível agravamento das doenças do aparelho circulatório pela TB ${ }^{19}$. Apesar de reconhecida a associação entre TB e diabetes, pelo agravamento da TB por este agravo ou vice-versa 20,21, não se observou relação na análise aqui realizada. Dessa forma, a formulação de uma estratégia de controle específica para essa comorbidade é necessária no país. Reis-Santos et al. 22 mostraram que indivíduos com TB e diabetes apresentavam outras comorbidades, como hipertensão arterial e doenças respiratórias, e tinham maior mortalidade do que indivíduos com TB sem diabetes. A desnutrição foi bastante presente, mas aconteceu igualmente nos dois períodos. Apesar de estudos apontarem sua associação com o óbito 23 , a desnutrição pareceu ser menos associada à precocidade do óbito e, talvez seja, um fator mais relacionado com o adoecimento por TB.
Óbitos sem menção da TB na DO que ocorreram no período de tratamento, em que a causa básica foi septicemia ou causas mal definidas e desconhecidas de mortalidade, possivelmente foram óbitos por TB, apontando para a baixa qualidade da informação e ausência de investigação e, consequentemente, comprometem a qualidade das estatísticas de morte 24,25,26. Visando a qualificar a informação das causas de morte no SIM, o Ministério da Saúde preconiza a investigação obrigatória de óbitos para grupos específicos (materno, infantil, mulher em idade fértil e causas mal definidas). Apesar dessa estratégia não ser executada para doenças específicas, sua implantação deve ser fortalecida nos serviços de saúde, dado os bons resultados alcançados no país 27 . O monitoramento sistemático e a presença de uma equipe altamente qualificada, aliados ao trabalho de campo constante, fazem com que a implantação e manutenção dessa atividade tenham alto custo, tornando mais difícil a adesão espontânea do setor saúde local. A recomendação de uma metodologia para investigação de óbitos pelo Programa Nacional de Controle da Tuberculose (PNCT) para a TB pode ser um primeiro passo. Por isso, a investigação desses óbitos é uma estratégia importante para esclarecimento da causa básica e para a melhoria da qualidade do SIM.

A inclusão da TB como uma das causas de morte nos 63 óbitos sem menção da doença na DO e com sobrevida menor que o período correspondente ao esquema básico de tratamento, representaria um acréscimo de $47,7 \%$ da TB como causa associada de óbito. Caso os 26 óbitos por causas presumíveis fossem confirmados na investigação, isto representaria um acréscimo de 13,7\% da TB como causa básica de óbito. Entretanto, supõe-se que esse número seja mais elevado, já que é frequente não haver resultado de exame de diagnóstico nos registros do SINAN. O aumento de óbitos por TB poderia ser observado também se considerássemos um período maior de sobrevida, como 270 dias, que corresponde ao limite de tempo para encerrar um caso tratado com esquema terapêutico básico mais 90 dias como limite de tempo para encerrar o caso ${ }^{6}$. Essa hipótese é reforçada pelo fato de que 27,4\% dos óbitos por causa básica TB ocorreram após 180 dias, ou seja, após o período de tratamento recomendado. Trata-se de uma proposta de investigação conservadora que precisa ser acompanhada e reavaliada quanto à sua efetividade, conforme a expertise adquirida no assunto.

As causas presumíveis de óbito por TB têm como objetivo direcionar e facilitar a investigação de óbitos, possibilitando resgatar óbitos ocorridos ainda durante o tratamento tera- 
pêutico básico, e que a TB possivelmente seria a causa básica.

As causas presumíveis sugeridas no presente trabalho concordam com outros estudos 28,29,30,31 que classificaram como óbito por TB aqueles com causa básica descritas como hemoptise; TB pulmonar progressiva sem insuficiência respiratória; TB meníngea; insuficiência respiratória sem outro motivo para tal; morte devido à resistência às medicações; efeitos adversos à medicação antiTB; uma combinação de infecção generalizada (sepse) com anemia, caquexia e morte súbita; embolia pulmonar; pneumonia; cor pulmonar.

A causa básica é amplamente utilizada na caracterização do diagnóstico da situação de saúde da população. Entretanto, conhecer as causas associadas possibilita dimensionar a magnitude de determinadas doenças, as quais têm sido ocultadas devido ao enfoque apenas na causa básica 32 . Laurenti \& Buchalla 33 também destacam que nem sempre a causa básica é uma doença ou evento simples de ser prevenido e, por isto, a análise das causas associadas pode ajudar na definição de ações preventivas.

Concluindo, os grupos considerados vulneráveis para a ocorrência de TB estão bem determinados (população de rua, privada de liberdade e TB/HIV), para os quais estratégias de controle específicas são recomendadas pelo Ministério da Saúde 9 . No entanto, com o crescimento da ocorrência de TB com a idade, a vigilância local deve atentar para a população idosa como um grupo populacional importante devido à associação com outras doenças crônicas e a maior ocorrência de eventos desfavoráveis.

Ainda que os transtornos mentais e comportamentais devido ao uso de álcool possam estar subnotificados, assim como os outros transtor- nos mentais (uso de drogas e psiquiátricos), a mortalidade dos pacientes com TB não parece ocorrer, prioritariamente, nestes grupos de extrema vulnerabilidade.

A coinfecção TB/HIV sugere que a dificuldade do diagnóstico precoce e a insuficiente atenção ofertada ao seguimento do tratamento têm desfavorecido a oportunidade de prevenir o desfecho óbito. A presença de 115 óbitos por AIDS sem menção da TB na DO demonstra que a coinfecção TB/HIV está subestimada nos registros de óbito. Considerando ainda que a AIDS ocorre em $80 \%$ das mortes como causa básica quando a TB é a causa associada e em $23 \%$ nas mortes sem menção da TB na DO, é fundamental que haja uma colaboração e envolvimento da vigilância epidemiológica da AIDS nesse processo.

O elevado percentual de óbitos ocorridos antes do término do tratamento terapêutico básico sem menção da TB na DO remete à descontinuidade do tratamento, um sério problema que ameaça o controle da TB no país. A barreira do acesso ao serviço e ao tratamento já havia sido vencida, restando a barreira à continuidade do cuidado, que deveria ser considerada prioridade pelos serviços. Por outro lado, o retardo no diagnóstico pode ter agravado o quadro clínico.

A vigilância de óbitos relacionados com a TB permitirá aumentar a completude dos sistemas de informação, elevar a proporção de casos encerrados, corrigir o SINAN e o SIM, resgatar os casos subnotificados e avaliar a qualidade do preenchimento do SIM 12,34. Todas essas medidas têm a função de melhorar a sensibilidade do sistema de vigilância e verificar a efetividade do tratamento. As razões pelas quais esse processo ainda não foi estabelecido plenamente devem ser ultrapassadas. 


\section{Resumen}

El objetivo fue analizar las múltiples causas de muerte en una cohorte de pacientes con tuberculosis (TB) y presentar una propuesta de investigación de las causas presumibles. Hicieron linkage probabilística entre el Sistema de Información de Enfermedades de Declaración Obligatoria (SINAN) 2006 y el Sistema de Información sobre Mortalidad (SIM) 2006-2008. 825 muertes, de las cuales $23 \%$ para TB, $16 \%$ con TB y $61 \%$ sin mención de TB. Doscientos quince $(42,7 \%)$ muertes ocurrieron antes del final de el tratamiento primario y tenía ninguna mención de TB, cuyo perfil era distinto del patrón observado cuando la TB era una causa asociada. La alta frecuencia de enfermedades respiratorias, SIDA y causas mal definidas sugieren insuficiencia en la calidad de la información. Hemos preparado propuestas de corrección de la causa asociada en el SIM y de investigación de muerte basado en las causas presumibles. De acuerdo con la propuesta, 26 muertes podrían haber modificado la causa subyacente. Destacase la gravedad de la TB y la importancia de el linkage para la vigilancia y la mejora de la información en el SIM y SINAN-TB.

Causas de Muerte; Tuberculosis; Mortalidad

\section{Colaboradores}

M. S. Rocha e R. S. Pinheiro participaram da concepção, análise, interpretação e redação do artigo. G. P. Oliveira, F. P. Aguiar e V. Saraceni contribuíram na análise, interpretação e redação do artigo.

\section{Agradecimentos}

Ao CNPq, Capes e FAPERJ pelo apoio financeiro.

\section{Referências}

1. World Health Organization. Global tuberculosis control: WHO Report 2011. Geneva: World Health Organization; 2011.

2. Fundação Nacional de Saúde. Manual de instruções para o preenchimento da declaração de óbito. 3a Ed. Brasília: Ministério da Saúde; 2001.

3. Santo AH. Causas múltiplas de morte relacionadas à tuberculose no Estado do Rio de Janeiro entre 1990 e 2001. J Bras Pneumol 2006; 32:544-52.

4. Drumond Jr. M. Vigilância da morte evitável: acesso rápido e descentralização das informações. In: Barreto ML, Almeida Filho N, Veras RP, Barata RB, organizadores. Epidemiologia, serviços e tecnologias em saúde. Rio de Janeiro: Editora Fiocruz/ Abrasco; 1998. p. 93-105. (Série EpidemioLógica, 3).
5. Laurenti R, Mello Jorge MHP, Gotlieb SLD. A mortalidade materna nas capitais brasileiras: algumas características e estimativa de um fator de ajuste. Rev Bras Epidemiol 2004; 7:449-60.

6. Ministério da Saúde. Sistema de Informação de Agravos de Notificação - SinanNET: manual do sistema. Brasília: Ministério da Saúde; 2007.

7. Coeli CM, Camargo Jr. KR. Avaliação de diferentes estratégias de blocagem no relacionamento probabilístico de registros. Rev Bras Epidemiol 2002; 5:185-96.

8. Santo AH, Pinheiro CE. Tabulador de causas múltiplas de morte. Rev Bras Epidemiol 1999; 2:90-7. 
9. Departamento de Vigilância Epidemiológica, Secretaria de Vigilância em Saúde, Ministério da Saúde. Manual de recomendações para o controle da tuberculose no Brasil. Brasília: Ministério da Saúde; 2011.

10. Selig L, Belo M, Cunha AJLA, Teixeira EG, Brito R, Luna $\mathrm{AL}$, et al. Óbitos atribuídos à tuberculose no Estado do Rio de Janeiro. J Bras Pneum 2004; 30:417-24.

11. Reis-Santos B, Gomes T, Macedo LR, Horta BL, Riley LW, Maciel EL. Prevalence and patterns of multimorbidity among tuberculosis patients in Brazil: a cross-sectional. Int J Equity Health 2013; 12:61.

12. Selig L, Kritski AL, Cascão AM, Braga JU, Carvalho RMG. Proposta de vigilância de óbitos por tuberculose em sistemas de informação. Rev Saúde Pública 2010; 44:1072-88.

13. Sousa LMO, Pinheiro RS. Óbitos e internações por tuberculose não notificados no Município do Rio de Janeiro. Rev Saúde Pública 2011; 45:31-9.

14. Oliveira GP, Pinheiro RS, Coeli CM, Barreira D, Codenotti SB. Uso do sistema de informação sobre mortalidade para identificar subnotificação de casos de tuberculose no Brasil. Rev Bras Epidemiol 2012; 15:468-77.

15. Jacomelli M, Silva PRAA, Rodrigues AJ, Demarzo SE, Seiscento M, Figueiredo VR. Broncoscopia no diagnóstico de tuberculose pulmonar em pacientes com baciloscopia de escarro negativa. J Bras Pneumol 2012; 38:167-73.

16. Instituto Nacional de Câncer José Alencar Gomes da Silva. Estimativa 2012: incidência de câncer no Brasil. Rio de Janeiro: Instituto Nacional de Câncer José Alencar Gomes da Silva; 2011.

17. Marcolino ABL, Nogueira JA, Ruffino-Netto A, Moraes RM, Sá LD, Villa TCS, et al. Avaliação do acesso às ações de controle da tuberculose no contexto das equipes de saúde da família de Bayeux - PB. Rev Bras Epidemiol 2009; 12:144-57.

18. Costa PV, Senna AL, Oliveira LGD, Siqueira RCAG, Figueiredo SP. Demora no diagnóstico da tuberculose pulmonar em cinco municípios da região metropolitana do Rio de Janeiro, Brasil, 2009-2010. Cad Saúde Colet (Rio J.) 2012; 20:195-202.

19. Sheu JJ, Chiou HY, Kang JH, Chen YH, Lin HC. Tuberculosis and the risk of ischemic stroke: a 3-year follow-up study. Stroke 2010; 41:244-9.

20. Dooley KE, Chaisson RE. Tuberculosis and diabetes mellitus: convergence of two epidemics. Lancet Infect Dis 2009; 9:737-46.

21. Dooley EK, Tang T, Golub JE, Dorman SE, Cronin W. Impact of diabetes mellitus on treatment outcomes of patients with active tuberculosis. Am J Trop Med Hyg 2009; 80:634-9.
22. Reis-Santos B, Locatelli R, Horta BL, Faerstein E, Sanchez MN, Riley LW, et al. Socio-demographic and clinical differences in subjects with tuberculosis with and without diabetes mellitus in Brazil - a multivariate analysis. PLoS One 2013; 8:e62604.

23. Waitt CJ, Squire SB. A systematic review of risk factors for death in adults during and after tuberculosis treatment. Int J Tuberc Lung Dis 2011; 15: 871-85.

24. Mello Jorge MHP, Gotlieb SLD, Laurenti R. O Sistema de Informações sobre Mortalidade: problemas e propostas para o seu enfrentamento. I - Mortes por causas naturais. Rev Bras Epidemiol 2002; 5:197-211.

25. Santo AH. Causas múltiplas de morte: formas de apresentação e métodos de análise [Tese de Doutorado]. São Paulo: Faculdade de Saúde Pública, Universidade de São Paulo; 1988.

26. Furukawa TS, Santo AH, Mathias TAF. Causas múltiplas de morte relacionadas às doenças cerebrovasculares no Estado do Paraná. Rev Bras Epidemiol 2011; 14:231-9.

27. Departamento de Análise de Situação em Saúde, Secretaria de Vigilância em Saúde, Ministério da Saúde. Saúde Brasil 2011: uma análise da situação de saúde e a vigilância de saúde da mulher. Brasília: Ministério da Saúde; 2012.

28. Davis Jr. CE, Carpenter JL, McAllister CK, Matthews J, Bush BA, Ognibene AJ. Tuberculosis. Cause of death in antibiotic era. Chest 1985; 88:726-9.

29. Ollé-Goig JE. Patients with tuberculosis in Bolivia: why do they die? Rev Panam Salud Pública 2000; 8:151-5.

30. Sack LV, Pendle S. Factors related to in-hospital death in patients with tuberculosis. Arch Intern Med 1998; 158:1916-22.

31. Mathew TA, Ovsyanikova TN, Shin SS, Gelmanova I, Balbuena DA, Atwood S, et al. Causes of death during tuberculosis treatment in Tomsk Oblast, Russia. Int J Tuberc Lung Dis 2006; 10:857-63.

32. Ishitani LH, França E. Uso das causas múltiplas de morte em Saúde Pública. Inf Epidemiol SUS 2001; 10:163-75.

33. Laurenti R, Buchalla CM. A elaboração de estatísticas de mortalidade segundo causas múltiplas. Rev Bras Epidemiol 2000; 3:21-8.

34. Sousa MGG, Andrade JRS, Dantas CF, Cardoso MD. Investigação de óbitos por tuberculose, ocorridos na Região Metropolitana do Recife (PE), registrados no Sistema de Informação de Mortalidade, entre 2001 e 2008. Cad Saúde Colet (Rio J.) 2012; 20:153-60.

Recebido em 07/Jul/2014

Versão final reapresentada em 29/Out/2014

Aprovado em 06/Nov/2014 